\title{
The Art of Surface Imaging
}

\author{
N.E. Piskunov \\ Astronomical Council of the USSR Academy of Sciences, Moscow, USSR \\ and \\ Observatory and Astrophysics Laboratory, University of Helsinki, Finland
}

\begin{abstract}
We intend to analyze the reliability of surface imaging of stars based on high resolution spectroscopy and the technique of inverse problem solution. Both astrophysical and mathematical aspects including different regularization methods are reviewed. The influence of the different factors on the resulting map is discussed and it is shown that the simultaneous use of different kinds of observational data (spectroscopy, photometry, polarimetry etc.) is very useful in providing additional constraints for the solution. The recent results in the surface imaging of $\mathrm{Cp}$ - and late-type stars show the way for further progress: the use of more adequate mathematical description of the stellar atmosphere and the simultaneous consideration of various surface inhomogeneities.
\end{abstract}

\section{Introduction}

The idea of the surface imaging of the stars was invented in 1958 by Deutsch who suggested using the Doppler shift produced by rotation in order to trace inhomogeneities on the surfaces of Ap-stars. He developed a method based on Fourier analysis (Deutsch, 1970) that in principle could provide information about the distribution of the spectral line intensity from its equivalent width variation as well as the changes in the apparent radial velocity. The subsequent modifications and improvements of the Deutsch method were made by Pyper (1969), Rice (1970), Falk and Wehlau (1974) and Mégessier (1975).

Further progress was made by Goncharskij and his collaborators (Goncharskij et al., 1977; Goncharskij et al., 1982; Goncharskij et al., 1983) which lead to the understanding that surface imaging is a fundamentally ill-posed problem. It has a multiplicity of solutions and any expansion method will provide just one of them but not necessarily the real distribution. Instead they proposed fitting the observed line profiles with the calculated ones by changing the surface distribution. The fitting is done according to a special mathematical procedure called the inverse problem solution that provides the unique solution.

Recently the surface imaging technique has been expanded to temperature mapping of the cool stars (Vogt and Penrod, 1982) and to magnetic field mapping of Cp-stars (Piskunov, 1985; Landstreet, 1988; Donati et al., 1990). Also, efforts have been made to replace the use of an approximation formula for the local line profile with the numerical solution of the transfer equation (Piskunov et al., 1990; Piskunov and Wehlau, 1990), to add different kinds of observational data, and to use several spectral lines simultaneously (Hackman et al., 1991). Several aspects of 
the surface imaging technique are discussed and illustrated by numerical examples in works by Piskunov (1985), Piskunov and Wehlau (1990), Vogt et al. (1987), Rice et al. (1989) and Semel (1989). An extensive reference list on the application of surface imaging technique to different stars is given in the paper by Khokhlova (1985).

\section{Mathematical formulation}

We have to establish a mathematical formulation of the problem of fitting observational data to the results of numerical simulation based on the theory of stellar atmosphere structure. Let $I(X(M), \lambda, \mu)$ be the specific intensity of the radiation with wavelength $\lambda$ emitted at point $M$ of the star surface in the direction $\mu$ (for strict definitions and notation see Mihalas, 1970). $X(M)$ represents a local characteristic of the point $M$ that determines the specific intensity $I$. It can be chemical element abundance, temperature, magnetic field, etc. The monochromatic flux at wavelength $\lambda$ will be given by a surface integral over the visible hemisphere:

$$
F_{\lambda}(\phi)=\iint I\left(X(M), \lambda+\Delta_{\lambda}, \mu\right) \mu d \sigma
$$

where $\phi$ is the rotational phase and $\Delta_{\lambda}$ - the Doppler shift due to the projected rotation velocity at the point $M$. If we can find a way to calculate $I(X(M), \lambda, \mu)$ for arbitrary set of parameters, we will be able to calculate fluxes for a given set of wavelengths and phases, and to compare them to the observational data. The function $X(M)$ that gives the best fit is the required map.

The inhomogeneous structure of the stellar surface produces the variations of $F_{\lambda}$ because of two different effects:

1) the contribution of any surface feature to the flux changes with the rotational phase because of limb darkening and the visibility of the feature;

2) the Doppler effect shifts the wavelengths of the contribution as the the projected velocity of the surface feature varies.

The difference between these two effects is that the first of them works independently of the rotation velocity of the star and can provide information about regions with small Doppler shifts. On the other hand, the second effect is much more pronounced in rapid rotators. Thus for producing a reliable image our simulation of line profiles must account correctly for both effects. To stress the importance of both effects we use the words Surface imaging rather than Doppler imaging, which was suggested by Vogt.

\subsection{Surface imaging as an inverse problem solution}

To compare the fluxes calculated from (1) with the observations we have to calculate the continuum level and allow for instrumental broadening. The continuum flux is expressed by:

$$
F_{\lambda}^{\text {cont }}(\phi)=\iint I^{\text {cont }}\left(X(M), \lambda+\Delta_{\lambda}, \mu\right) \mu d \sigma
$$


where $I^{\text {cont }}(X(M), \lambda, \mu)$ is the continuum specific intensity. The dependence of $I$ and $I^{\text {cont }}$ on $X$ can be very different. For example, if $X(M)$ represents the abundance of a rare-earth element at point $M, I^{\text {cont }}$ will not depend on $X$ at all. The Doppler shifts can be neglected, while calculating $F^{\text {cont }}$.

Now $F_{\lambda}$ must be convoluted with the instrumental profile, usually approximated by a Gaussian:

$$
\mathcal{F}_{\lambda}(\phi)=\frac{1}{\sqrt{\pi} \Delta \lambda_{\text {instr }}} \cdot \int_{-\infty}^{\infty} e^{-\left(\frac{y-\lambda}{\Delta \lambda_{\text {instr }}}\right)^{2}} F_{y}(\phi) d y .
$$

$\Delta \lambda_{\text {instr }}$ is the half width of the instrumental profile. So finally we calculate the residual intensity $r_{\lambda}$ :

$$
r_{\lambda}(\phi)=\frac{\mathcal{F}_{\lambda}}{F^{\text {cont }}}
$$

and the surface imaging problem becomes mathematically equivalent to searching for such $X(M)$ that provides the minimum of the discrepancy function $D$ :

$$
D(X)=\sum_{\phi, \lambda} \omega_{\phi \lambda} \cdot \frac{\left(r_{\lambda}(\phi)-r_{\lambda}^{\mathrm{obs}}(\phi)\right)^{2}}{N_{\phi} N_{\lambda}}
$$

where summing is performed over all available rotational phases and wavelengths. The weights $\omega_{\phi \lambda}$ are meant to account for the possible differences in the observational errors for each $r_{\lambda}^{o b s}(\phi)$. If $\sigma_{\phi \lambda}$ is the observational error and $\sigma_{\min }$ is the minimum of all errors, the weights can be defined as $\omega_{\phi \lambda}=\sigma_{\min } / \sigma_{\phi \lambda}$. The problem (5) is known as the inverse problem. The name comes from the fact that if $D$ is formally treated as an operator over $X$, the solution of (5) can be obtained by constructing the inverse operator $D^{-1}$. But the very bad behaviour of $D^{-1}$ means that the problem (5) is a so-called ill-posed problem (for strict definition see Tikhonov, 1963; Shore and Johnson, 1980). The major consequence of this is the multiplicity of solutions of (5). The theory to treat ill-posed problems was developed in the early 1960 's, but it has only recently found its practical applications. The method is known as a regularization method.

\subsection{Regularization method}

The idea of the regularization is to replace the problem (5) with another one with better properties and a unique solution, which approximates in a certain known way the real $X(M)$. We shall assume now that $X(M)$ is a scalar function so that the mapping is performed just for one surface characteristic, e.g. temperature. The case of vector $X(M)$ is discussed in sections 6 and 7 . The regularized problem can be expressed in the form:

$$
\Phi(X)=D(X)+\Lambda \cdot R(X)
$$


where $\Lambda$ is a conventional Lagrange multiplier. $R(X)$ is the regularization functional which implies some additional constraints on the possible solution and thus makes it unique. Problem (6) can be considered as a typical conditional minimization problem. The value of $\Lambda$ should be selected in such a way that if $X(M)$ is the solution of $(6)$ then

$$
D(X)=\sigma_{\text {obs }},
$$

where $\sigma_{\text {obs }}$ is the mean observational error (for the corresponding theorems see Tikhonov, 1963; Yagola, 1979; Shore and Johnson, 1980), so if the quality of the observations is better, the influence of the regularization $R(X)$ is smaller and we are getting closer to the solution of (5) realized in Nature.

Several forms of the regularization function are known. Two of them are quite general: the Tikhonov function and the entropy measure. Others have a more limited range of application and are based on additional information about the physical processes that control $X(M)$ (for examples, see Goncharskij and Stepanov, 1979).

The Tikhonov regularization function

$$
R^{T}(X)=\iint\|\nabla X(M)\|^{2} d \sigma
$$

is a measure of a smoothness of $X(M)$ so that the resulting map provides the smoothest possible solution that is able to reproduce the observations within the observational errors. The use of (8) as the regularization function for (6) means that a strong correlation between neighbouring points is assumed.

The Entropy regularization function is used in the form

$$
R^{E}(X)=\iint X(M) \log X(M) d \sigma
$$

and assumes no correlation between neighbouring points.

Although the Tikhonov function seems to be more appropriate for the surface imaging of the stars, both (8) and (9) can be used because, as can be seen from Fig. 1, with high quality data the rôle of the regularization function is limited to providing the unique solution and is small compared to the influence of the observations. More detailed discussion of the two methods can be found in papers by Khokhlova et al. (1986) and Piskunov et al. (1990).

\subsection{How close can we get to reality?}

According to the theorems mentioned above, the inverse problem solution will converge to the exact surface distribution of $X(M)$ as the quantity and the accuracy of the data increase. But while our observations are not perfect we still can get a unique solution which approximates the real surface distribution.

Also with both the Tikhonov and MEM regularizations it is difficult to say what is the upper limit for the differences between the calculated and the measured $X(M)$ all over the stellar surface. Several indirect approaches can be suggested. For example, comparison of the abundance maps, derived from the data for different 

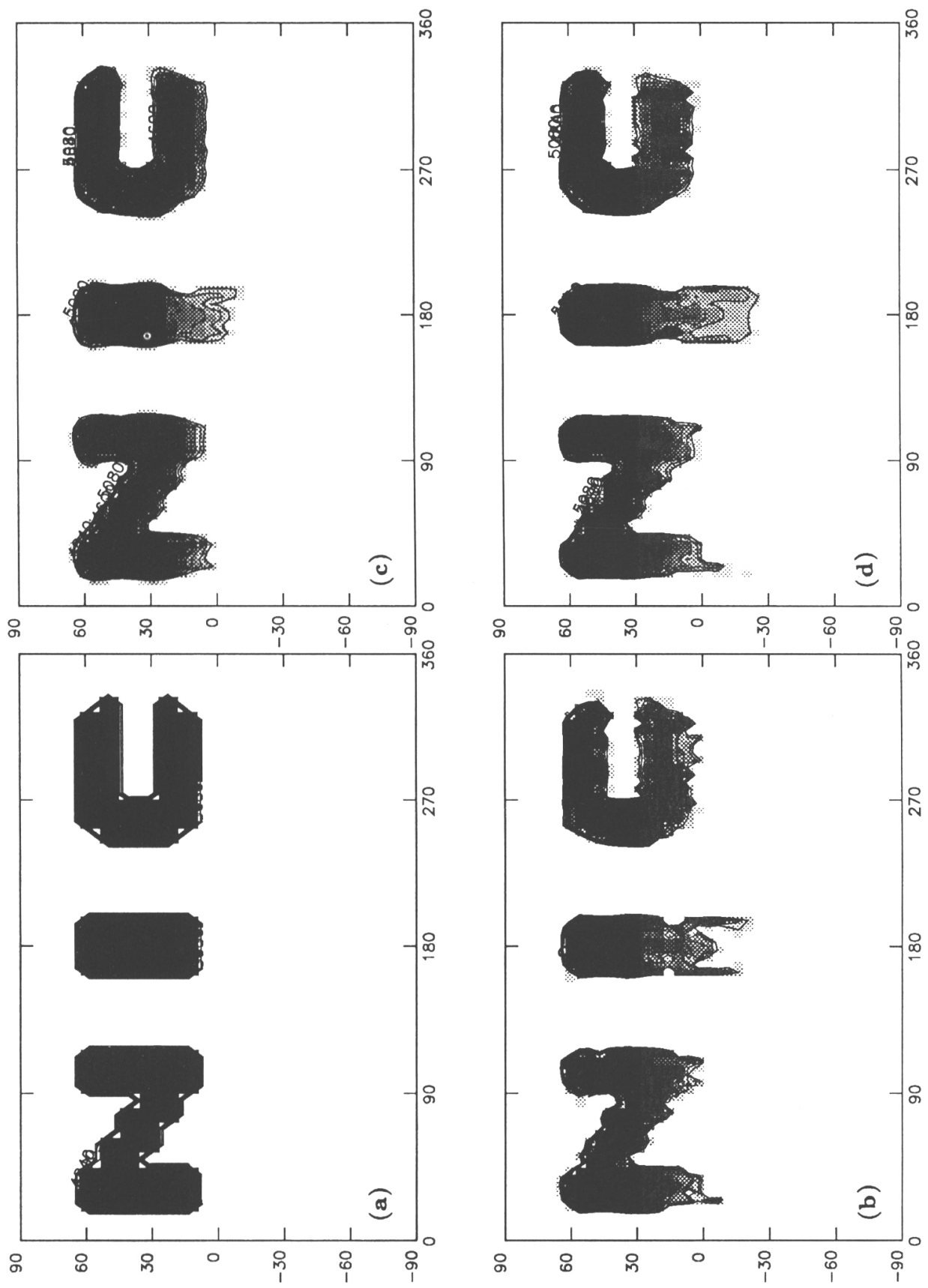

Fig. 1. A comparison of the inverse problem solutions for NIC-star (a) without regularization (b), and using Tikhonov (c) and Maximum entropy (d) regularizations. S/N is 500. Spectral resolution 60000 . Temperature inside the letters is $1500 \mathrm{~K}$ lower than on the rest of the surface. Ca I $\lambda 6439.1$ line profiles were used for the imaging. 
spectral lines of a given element, might be used for Cp-stars. For late type stars the comparison of the different kinds of observations with the simulations based on the obtained maps, such as photometric light curves, can provided an estimate of the error.

\section{The resulting map and the observational data parameters}

The quality and the quantity of the spectral observations influence the produced image in different ways. All parameters of the observations (spectral resolution, $\mathrm{S} / \mathrm{N}$ ratio, and phase coverage) are important for correct mapping. We are going to illustrate an influence of all major characteristics of the observational data using the numerical simulations of 25 profiles of $\mathrm{Ca}$ I $\lambda 6439.1$ line for the artificial "NIC-star" shown on Fig. 1a.

\subsection{Spectral resolution}

The spectral resolution should be consistent with the $S / N$ ratio and the rotational broadening of the spectral lines. This balance also determines the surface grid size (and thus the size of the surface resolution) that it is reasonable to use for a given star (for detailed discussion see Piskunov and Wehlau, 1990). Figure 2 shows the result of NIC-star reconstruction with a reduced spectral resolution.

\subsection{Signal-to-noise ratio}

The signal-to-noise ratio determines how accurate the amplitude of the surface feature can be reconstructed. Numerical experiments prove that $S / N=150$ is the lower limit for the observational data to be used in surface imaging. High $\mathrm{S} / \mathrm{N}$ ratio is also necessary to take advantage of a high spectral resolution (if any), especially for rapidly rotating stars where the slope of the line wings is small. Figure 3 shows the map of the NIC-star obtained from the line profiles with the simulated $\mathrm{S} / \mathrm{N}=$ 100 .

\subsection{Phase coverage}

The phase distribution of the observations is supposed to cover all of the stellar surface visible from the Earth. If some longitudes are not represented in any of the line profiles, the whole solution will be quite unstable because the assumption of the continuity of the surface distribution is not anymore applicable. The computer code usually keeps the initial distribution inside a gap in longitude coverage but this is of course a purely artificial decision.

Good phase coverage is important not only for achieving a good surface resolution (smaller features will manifest themselves at many different Doppler shifts) but also for differentiating between north and south hemispheres. The surface points below and above the equator can be distinguished from each other (for intermediate values of the inclinations of the star rotation axis) only because of the difference in their times of visibility during the rotational period, and the difference in the amplitudes of the Doppler shift variations. Figure 4 illustrates the reconstruction of the NIC-star from data for phases between 0 and 0.75 only. 


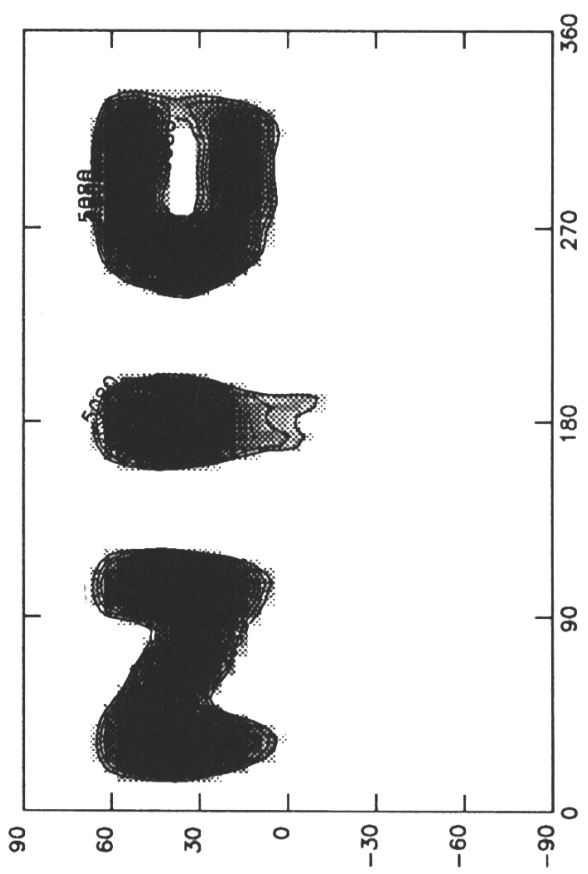

Fig. 2. The results of the NIC-star mapping using the line profiles with spectral resolution 20000 .

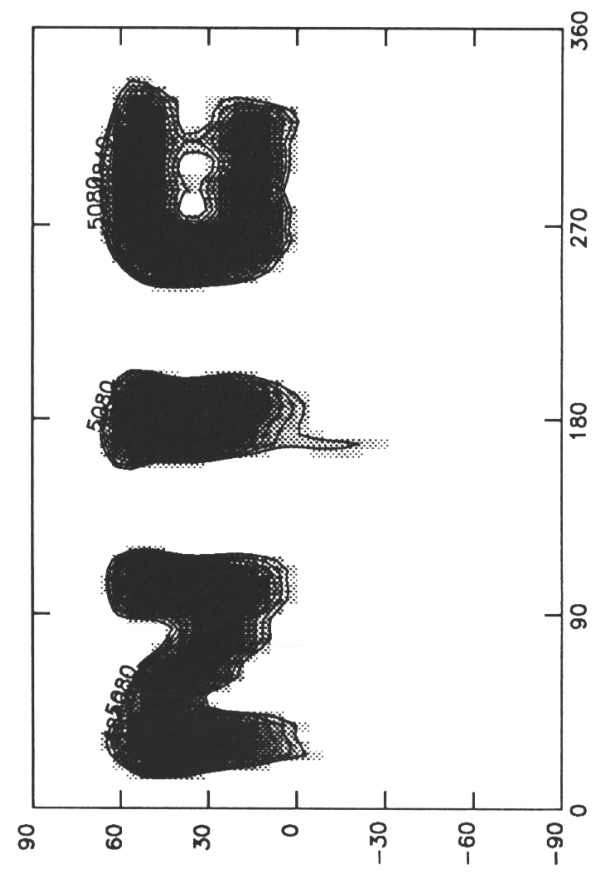

Fig. 3. The results of the NIC-star mapping using the line profiles with $\mathrm{S} / \mathrm{N}=$ 100.

\section{The resulting map and the stellar characteristics}

Knowledge of the stellar rotation velocity and the inclination angle is necessary to start the inverse problem solution. In some cases the accuracy of those characteristics can be improved during the imaging.

\subsection{Rotation velocity}

Because only rapidly rotating stars are selected for surface imaging, the line profiles are dominated by the rotational broadening, so that the procedure of fitting the observations with the calculated profiles is very sensitive to the error in $V \sin i$. It is impossible to get the discrepancy function down to the observational errors if $V \sin i$ is more than $10 \mathrm{~km} / \mathrm{s}$ away from its real value. Smaller errors of the order of $5 \mathrm{~km} / \mathrm{s}$ may result in an artificial equatorial belt of enhanced local line strength if the assumed velocity is too low, or in the polar cap if the assumed velocity is too high. Those features are quite helpful for fine tuning of the rotation velocity value. But it is very difficult to achieve an accuracy better than $\pm 2 \mathrm{~km} / \mathrm{s}$ (see 
Vogt et al., 1987). An attempt to map the NIC-star with an incorrect $V \sin i$ is illustrated in Fig. 5.

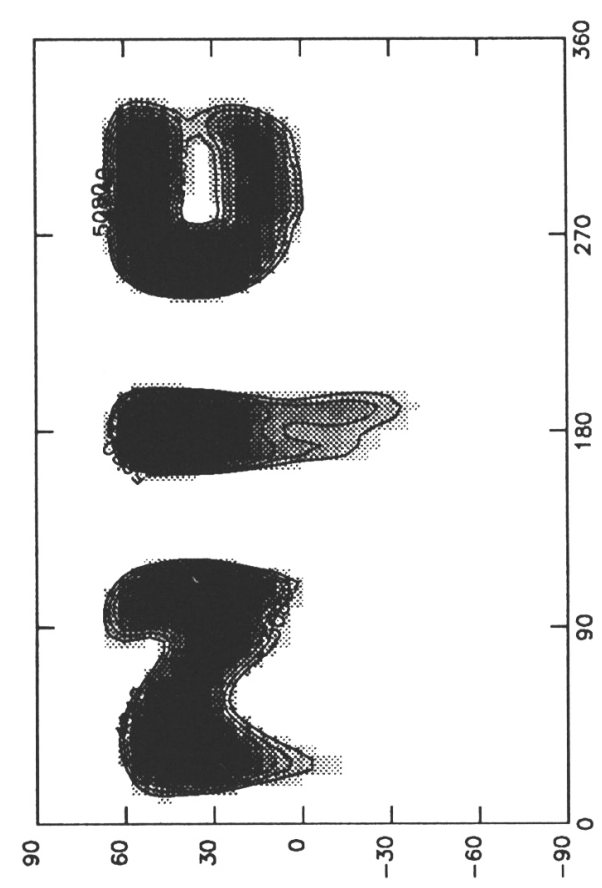

Fig. 4. The results of the NIC-star mapping using line profiles for phases between 0 and 0.75 .

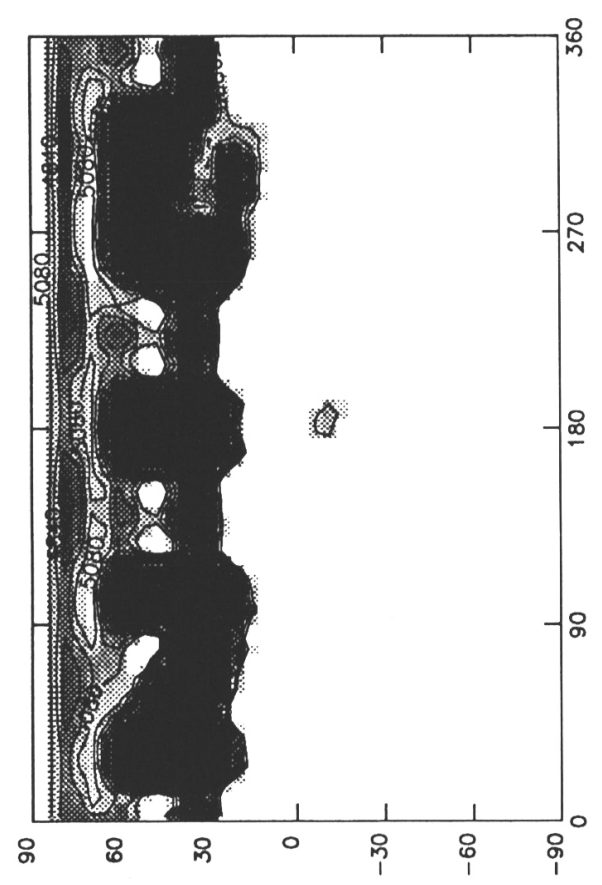

Fig. 5. The results of the NIC-star mapping using $V \sin i=53 \mathrm{~km} / \mathrm{s}$ instead of $55 \mathrm{~km} / \mathrm{s}$.

\subsection{Inclination}

The influence of the inclination angle is not so strong as that of the rotation velocity. Changing the value of $i$ can move the surface features to a different latitudes while the shape and the amplitude of the spots remain essentially the same (see Khokhlova et al., 1986). The required accuracy for $i$ is about $\pm 10^{\circ}$.

\section{The resulting map and the line profile calculation}

The quality of the specific intensity calculations used for high $\mathrm{S} / \mathrm{N}$ data simulation is a crucial point for the extraction of the information about the surface structure. A poor approximation for $I(X, \lambda, \mu)$ may produce some artifacts in the resulting map. Now we shall see how different methods treat the following six problems of major importance for surface imaging:

1) line parameter selection (oscillator strength and line broadening constants); 
2) model atmosphere selection (numerical or simplified, e.g. Milne-Eddington);

3) accounting for line shape variation;

4) treatment of blends;

5) accuracy of the representation of the line profiles;

6 ) interpretation of the resulting maps.

\subsection{Using the approximation formula}

An approximation formula for the surface profile of the specific intensity $I(X, \lambda, \mu)$ was generally used by many researchers because it is much faster to compute and it is usually expressed in analytical form, so that the accurate calculation of gradients is much easier. Several formulae were used: Minnaert function (Goncharskij et al., 1977), Unno functions (Piskunov and Khokhlova, 1983), etc.

When using an approximation formula problems 1) and 2) are replaced by the problem of the correct selection of the formula parameters. This selection can be made by comparing the approximation formula with more accurate calculations for a wide set of atmosphere conditions, although for this calculation problem 1) still exists (Khokhlova and Pavlova, 1984). Problem 3) is sometimes neglected (Vogt et al., 1987) but can be accounted for if a separate approximation is used for the the line and the continuum.

The approximation formula approach has nowadays mainly historical interest because:

- it is unable to deal with high $\mathrm{S} / \mathrm{N}$ observations (the approximation accuracy is usually between 1 and 2 percent);

- it is practically impossible to treat blends;

- the interpretation of the results is not straightforward because we usually get maps of approximation formula parameters and an extra step is needed to obtain physical parameter distributions over the surface.

\subsection{Transfer equation solution}

The explicit solution of the transfer equation reproduces the observed data in a much more satisfactory manner, accounts for continuum variation and can be easily extended to treat blends (see 5.3). Also, no additional interpretation of the results is required. Advanced numerical model atmospheres and even several different models for different surface points can be used, although the problem of obtaining a reliable model atmosphere still exists. The numerical solution of the transfer equation must be done many times for all surface points, so it is worthwhile to pre-calculate the interpolation tables for specific intensity in order to reduce the computing time. One possible approach that we are currently using (Piskunov et al., 1990; Piskunov and Wehlau, 1990) requires the calculation of two tables. The first is wavelength dependent and contains $I(X, \lambda, \mu)$ values for a set of nodes over all three parameters. The second stores the continuum specific intensities and does not depend upon the wavelength. Storing tables for the values of $\mu$ used for surface integration reduces the calculation of the local profile to a 2-dimensional interpolation (if $X(M)$ is a scalar function, such as in the cases of abundance or temperature mapping). 


\subsection{Spectral synthesis}

The use of spectral synthesis is a further development of the surface imaging technique based on the numerical solution of the radiation transfer equation. The spectral synthesis is not simply a tool for the correct treatment of spectral blends but also provides the additional constraints for the inverse problem solution. The reliability of the resulting image can be significantly improved by a simultaneous fitting of a set of spectral lines with a different sensitivity to the mapping parameter. This possibility was first noticed by Landstreet (1988) and he successfully used it for the imaging of the magnetic field on $53 \mathrm{Cam}$, selecting lines with different Landé factors. The results of temperature mapping of EI Eri, using a spectral synthesis technique for a blend of five lines, is presented by Hackman et al., in these Proceedings.

\section{Using additional data}

Using a combination of different kinds of observational data can be very helpful in improving the reliability and the quality of the imaging. It can also provide information about star geometry (orientation of the rotation axis), magnetic field strength and distribution, etc.

\subsection{Photometry}

When mapping the temperature on the surface of late-type stars the photometry provides an important test for the correct regularization of the solution. Too small a $\Lambda$ value will result in extra temperature spots on the surface which will distort the light curves. The transfer equation (or spectral synthesis) method of line profile simulation can be easily checked for consistency with photometric data by producing a temperature map from spectral line(s) only, calculating the emerging flux for a set of rotation phases and convoluting it with a corresponding filter functions. The results can be compared with the photometric light curves. An example of this kind of comparison for EI Eri is shown in Hackman et al.(these Proceedings). We want also to stress here the importance of a good knowledge of the rotational period as well as the use of simultaneous spectroscopy and photometry for late-type stars with strong surface activity.

\subsection{Polarization}

The achievements from using polarization observations for surface imaging are rather modest. For $\mathrm{Cp}$-stars, only some restricted multipole expansion approximation for magnetic field have been considered (e.g. Borra and Landstreet, 1978; Glagolevskij et al., 1985; Donati et al., 1990).

The big challenge when using polarization observations for the surface imaging is that this kind of data is much more sensitive to the surface structure than the line profiles. According to the numerical modelling (Piskunov and Khokhlova, 1983, 1984; Semel, 1989) with the polarization data it may be possible to map polar region and to break symmetry in the equatorial belt. For late-type stars it may provide a unique opportunity to measure the field strength inside the active regions. 


\subsection{Several spectral regions}

Additional information can also be obtained simply by using several spectral regions with different properties and fitting them simultaneously. When mapping temperature in this way one can select lines with different temperature sensitivity belonging to different ionization stages. For $\mathrm{Cp}$-stars this method provides a possibility of increasing the amount of observational data or of mapping the distribution of several chemical elements (if $X(M)$ is taken in vector form).

Landstreet (1988) suggested using several spectral regions for magnetic stars in order to extract information about the surface field by fitting the intensification of lines as well as the distances between Zeeman components of lines with different Landé factors.

\section{Conclusions}

Surface imaging has become an important tool for studying individual stars with strong surface activity. Further improvement of the quality of the spectroscopic observations and in the understanding by the observers of the requirements of surface imaging will provide possibilities for new kinds of research, for example, studying the evolution of the surface features in late-type stars or making a statistical analysis of elements and magnetic field distribution in Cp-stars.

To meet these approaching opportunities efforts should be directed towards determining better spectral line data, and producing better atomic data, atmosphere models, etc.

The implementation of spectral synthesis techniques, together with the use of several kinds of observations, will enable us to produce the most reliable and detailed maps.

High quality polarization observations are the only way to study the magnetic field on the surfaces of rapidly rotating stars.

\section{References}

Borra, E.F., Landstreet, J.D.: 1978, Astrophys. J. 222, 226

Deutsch, A.: 1958, in Electromagnetic Phenomena in Cosmological Physics, IAU Symp. 6, ed. B. Lehnert, Cambridge Univ. Press, Cambridge, p. 209

Deutsch, A.: 1970, Astrophys. J. 159, 895

Donati, J.-F., Semel, M., del Toro Iniestia, J.C.: 1990, Astron. Astrophys. Lett. 233, L17

Falk, A.F., Wehlau, W.H.: 1974, Astrophys. J. 192, 409

Glagolevskij, Yu.V., Piskunov, N.E., Khokhlova, V.L.: 1985, Sov. Astron. Lett. 10, 187

Goncharskij, A.V., Stepanov, V.V.: 1979, Sov. Math. Dokl. 20, 414

Goncharskij, A.V., Stepanov, V.V., Khokhlova, V.L., Yagola, A.G.: 1977, Sov. Astron. Lett. 3, 147

Goncharskij, A.V., Stepanov, V.V., Khokhlova, V.L., Yagola, A.G.: 1982, Sov. Astron. 19, 576

Goncharskij, A.V., Ryabchikova, T.A., Stepanov, V.V., Khokhlova, V.L., Yagola, A.G.: 1983, Sov. Astron. 27, 49 
Khokhlova, V.L.: 1985, Astrophys. Space Phys. Rev., Soviet Scientific Rev., Sect. E., ed. R. Sunyaev, 4, p. 99

Khokhlova, V.L., Pavlova V.M: 1984, Sov. Astron. Lett. 10, 158

Khokhlova, V.L., Rice, J.B., Wehlau, W.H.: 1986, Astrophys. J. 307, 768

Landstreet, J.D.: 1988, Astrophys. J. 326, 967

Mégessier, C.: 1975, Astron. Astrophys. 39, 263

Mihalas, D.: 1970, Stellar Atmospheres, Freeman, San Francisco

Piskunov, N.E.: 1985, Sov. Astron. Lett. 11, 18

Piskunov, N.E., Khokhlova, V.L.: 1983, Sov. Astron. Lett. 9, 346

Piskunov, N.E., Khokhlova, V.L.: 1984, Sov. Astron. Lett. 10, 187

Piskunov, N.E., Tuominen, I., Vilhu, O.: 1990, Astron. Astrophys. 230, 363

Piskunov, N.E., Wehlau, W.H.: 1990, Astron. Astrophys. 233, 497

Pyper, D.: 1969, Astrophys. J. Suppl. 18, 347

Rice, J.B.: 1970, Astron. Astrophys. 9, 189

Rice, J.B., Wehlau, W.H., Khokhlova, V.L.: 1989, Astron. Astrophys. 208, 179

Semel, M.: 1989, Astron. Astrophys. 225, 456

Shore, J.E., Johnson, R.W.: 1980, IEEE Trans. IT-26, 26

Tikhonov, A.N.: 1963, Sov. Math. Dokl. 4, 1624

Vogt, S.S., Penrod, G.D.: 1982, in Activity in Red-Dwarf Stars, IAU Coll. 71, eds. P. Byrne, M. Rodonò, Reidel, Dordrecht, p.379

Vogt, S.S., Penrod, G.D., Hatzes, A.P.: 1987, Astrophys. J. 321, 496

Yagola, A.G.: 1979, Sov. Math. Dokl. 20, 275 